\title{
Surface anchoring on layers of grafted liquid-crystalline chain molecules: A computer simulation
}

\author{
Harald Lange ${ }^{\dagger, \ddagger}$ and Friederike Schmid ${ }^{\dagger}$ \\ $\dagger$ Fakultät für Physik, Universität Bielefeld, 33615 Bielefeld, Germany \\ † Institut für Physik, Universität Mainz, 55099 Mainz, Germany
}

\begin{abstract}
By Monte Carlo simulations of a soft ellipsoid model for liquid crystals, we study whether a layer of grafted liquid-crystalline chain molecules can induce tilt in a nematic fluid. The chains are fairly short (four monomers) and made of the same particles as the solvent. They are attached to a substrate which favors parallel (planar) alignment. At low grafting densities, the substrate dominates and we observe planar alignment. On increasing the grafting density, we find a first order transition from planar to tilted alignment. Beyond the transition, the tilt angle with respect to the surface normal decreases continuously. The range of accessible anchoring angles is quite large.
\end{abstract}

PACS numbers: 61.30.Hn, 61.30.Vx

\section{INTRODUCTION}

Surfaces orient nematic liquid crystals, because they align nearby particles 3 3. This phenomenon, called surface anchoring, is net only interesting from a fundamental point of view 6 ; it also plays a key role in liquid crystal display devicest. 6 . Surface modifications which allow to tune the anchoring properties of a surface - in particular the anchoring angle - have great practical importances 10 .

To be useful in technological applications, such modified surfaces must be physically and chemically stable. Therefore, preparation techniques where molecules are attached chemically to the surface are desirable. The coupling between the surface and the nematic fluid must be well-defined, i. e., strong. This calls for the use of large molecules (polymers), which have themselves liquid crystalline properties.

Based on such considerations, Peng, Johannsmann and Rühe have recently investigated the anchoring behavior of nematic liquid crystals on thers of grafted side-chain liquid crystalline polymers 11 14 1 "grafting-from" technique developed by Rühe et al15.16 allowed to synthesize brushes of up to $230 \mathrm{~nm}$ dry thickness. They were forced to grow into a homogeneously aligned structure by an appropriate treatment of the substrate prior to the grafting step. When brought in contact with a nematic fluid, they transfered their orientation into the fluid13. Thus Peng et al have demonstrated experimentally that surfaces with grafted liquid crystalline chain molecules can align nematic liquid crystals.

The idea of using liquid crystalline polymer brushes as alignment layers goes back to Halperin and Williams 19 . They suggested to create a competition between the orienting effect of the bare substrate, and that of stretched chains. The anchoring angle should then be determined by the properties of the substrate at low grafting densities, and by the chain stretching at high grafting densities. Simple mean field considerations lead Halperin and Williams to the conclusion that the two regimes should be separated by a second order transition. This implies that arbitrary anchoring angles can be realized at intermediate grafting densities.

The actual calculation of Halperin and Williams relies on two assumptions: The chains are very long and can be treated as entropic springs, and they are swollen by a good nematic solvent of low molecular weight. At least the second condition is not met in real systems: The brushes of Peng et al were only swollen to a very limited extent, even in a chemically similar solvent. Nevertheless, the general idea still seems worth pursuing: Consider a substrate which is decorated with liquid-crystalline chain molecules (main-chain liquid-crystals for simplicity), and which favors parallel alignment: Without grafted chains, the substrate aligns the fluid in a planar way. At high grafting densities, the chain molecules are forced to stand up. The question is: What happens at intermediate densities?

The high grafting density limit has been discussed in an earlier paper 20 for the case of short chains in a moderate solvent. Based on a simple theoretical model, we have conjectured that there exists a continuous anchoring transition from tilted to perpendicular anchoring, which is driven by the grafting density. The theory predicts that the anchoring angle (with respect to the surface normal) is always smaller than the tilt angle inside of the chain layer. Thus the anchoring transition in the bulk preempts the corresponding tilting transition in the chain layer, and may even exist if there is no such tilting transition. Computer simulations were consistent with that picture, though not conclusive. This anchoring transition has nothing to do with the transition predicted by Halperin and Williams, which separates a phase with planar alignment from a phase with tilted alignment. 
From a practical point of view, the low grafting density regime is more important, since low grafting densities can be realized much more easily in experiments. In the present paper, we study the anchoring behavior at low and intermediate grafting densities by Monte Carlo simulations of an idealized, coarse-grained model. The general setup of the model is such that, strictly speaking, the Halperin/Williams theory does not apply: The chains are much too short. Nevertheless, we will see that the anchoring transition persists - now as a weakly first oder transition. Our results demonstrate that the scenario predicted by Halperin and Williams is valid in a much wider range of parameters than that for which it was derived originally.

In the next section, we introduce the model and describe the simulation method. The results are presented in section III. We summarize and discuss our findings in section IV.

\section{MODEL AND METHOD}

In our model, both the chain monomers and the solvent particles are represented by soft ellipsoidal particles with elongation $\kappa=\sigma_{\text {end-end }} / \sigma_{\text {side-side }}=3$. Two particles $i$ and $j$ with orientations $\mathbf{u}_{i}$ and $\mathbf{u}_{j}(|\mathbf{u}|=1)$ at the positions $\mathbf{r}_{i}$ and $\mathbf{r}_{j}$ interact with the pair potential

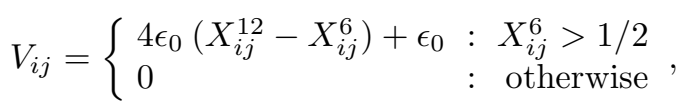

where $X_{i j}=\sigma_{0} /\left(r_{i j}-\sigma_{i j}+\sigma_{0}\right)$ depends on the centercenter distance $r_{i j}=\left|\mathbf{r}_{i}-\mathbf{r}_{j}\right|$ and the contact distance $\sigma_{i j}$ of two ellipsoids in the direction $\hat{\mathbf{r}}_{i j}=\left(\mathbf{r}_{i}-\mathbf{r}_{j}\right) / r_{i j}$. We approximate the latter by the gaussian overlap function suggested by Berne and Pechukas:

$$
\begin{aligned}
& \sigma_{i j}\left(\mathbf{u}_{i}, \mathbf{u}_{j}, \hat{\mathbf{r}}_{i j}\right)=\sigma_{0}\left\{1-\frac{\chi}{2}\right. \\
& \left.\quad\left[\frac{\left(\mathbf{u}_{i} \cdot \hat{\mathbf{r}}_{i j}+\mathbf{u}_{j} \cdot \hat{\mathbf{r}}_{i j}\right)^{2}}{1+\chi \mathbf{u}_{i} \cdot \mathbf{u}_{j}}+\frac{\left(\mathbf{u}_{i} \cdot \hat{\mathbf{r}}_{i j}-\mathbf{u}_{j} \cdot \hat{\mathbf{r}}_{i j}\right)^{2}}{1-\chi \mathbf{u}_{i} \cdot \mathbf{u}_{j}}\right]\right\}^{-1 / 2}
\end{aligned}
$$

with $\chi=\left(\kappa^{2}-1\right) /\left(\kappa^{2}+1\right)$. The interactions (1) are identical for solvent particles and chain monomers. In addition, chain monomers are connected by bonds of length $b$, which are subject to a spring potential with an equilibrium length $b=b_{0}$ and a logarithmic cutoff at $\left|b-b_{0}\right|=b_{s}$.

$$
V_{S}(b)= \begin{cases}-\frac{k_{s}}{2} b_{s}^{2} \ln \left(1-\frac{\left(b-b_{0}\right)^{2}}{b_{s}^{2}}\right) & :\left|b-b_{0}\right|<b_{s} \\ \infty & :\left|b-b_{0}\right|>b_{s}\end{cases}
$$

The chains are stiff by virtue of a stiffness potential

$$
V_{A}\left(\theta_{1}, \theta_{2}, \theta_{12}\right)=-k_{a}\left[\cos \left(\theta_{1}\right)+\cos \left(\theta_{2}\right)+2 \cos \left(\theta_{12}\right)\right] \text {, }
$$

which depends on the angles $\theta_{1}$ and $\theta_{2}$ between the orientation of a monomer and the adjacent bonds, and the angle $\theta_{12}$ between the two bonds.

The system is confined between two hard walls at $z=0$ and $z=L_{z}$. A hard core potential prevents the particles from penetrating the walls,

$$
\begin{gathered}
V_{W}(z)=\left\{\begin{array}{lll}
0 & : & d_{z}(\theta)<z<L_{z}-d_{z}(\theta) \\
\infty & : & \text { otherwise }
\end{array},\right. \\
d_{z}(\theta)=\sigma_{0} / 2 \sqrt{1+\cos ^{2}(\theta)\left(\kappa^{2}-1\right)} .
\end{gathered}
$$

The function $d_{z}(\theta)$ is the contact distance between a surface and an ellipsoid of diameter $\sigma_{0}$ and elongation $\kappa$, with the long axis oriented at the angle $\theta$ with respect to the surface normal. The first bond of each chain is attached to one of the surfaces. The grafting points are on a regular square lattice. The grafting is fully flexible, i. e., no energy contribution couples to the angle between the first bond and the surface normal.

We chose the model parameters $k_{s}=10 \epsilon_{0} / \sigma_{0}^{2}, k_{a}=$ $10 \epsilon_{0}, b_{0}=4 \sigma_{0}$ and $b_{s}=0.8 \sigma_{0}$. The simulations were performed at the temperature $T=0.5 \epsilon_{0} / k_{B}$ and pressure $P=3 \epsilon_{0} / \sigma_{0}^{3}$. This corresponds to a state well in the nematic phase: The transition to the isotropic phase occurs at the pressure21, $22 P=2.3 \epsilon_{0} / \sigma_{0}^{3}$. The bulk number density was $\langle\rho\rangle=0.313 / \sigma_{0}^{3}$. The length of the chains was four bonds, (i. e., four monomers plus the grafting point). They are very stiff: The persistence length is roughly $210 \sigma_{0}$, and the end-to-end distance of free chains is $14.8 \sigma_{0}$. Compared to experimental systems, our chains correspond to main chain oligomers with very few (less than ten) mesogenic units. We have not attempted any quantitative mapping on a specific system.

The simulations were conducted in the NPT ensemble, at constant temperature and pressure, and a fixed number of particles. The systems contained roughly 2000 solvent particles (the numbers varied slightly in the different runs) and a varying number of chains with four monomers. The simulation boxes were rectangular with fixed lateral size $L_{\|}$and fluctuating length $L_{z}$. The lateral size was kept fixed in order to maintain a constant grafting density $\Sigma$. It was chosen $L_{\|}=12 \sigma_{0}$ in most runs, except for four runs which sampled grafting densities between $\Sigma=0.111 / \sigma_{0}^{2}(4 \times 4$ chains on an area $\left.12 \cdot 12 \sigma_{0}^{2}\right)$ and $\Sigma=0.174 / \sigma_{0}^{2}(5 \times 5$ chains $)$. These were conducted in systems with $5 \times 5$ chains and slightly larger surface areas. The length $L_{z}$ of the boxes was roughly $L_{z} \approx 45-64 \sigma_{0}\left( \pm 10 \sigma_{0}\right)$ (higher for higher grafting density. Monte Carlo moves were carried out in random order and included particle displacements, particle rotations, and rescaling of the simulation box in the $z$ direction. They were accepted or rejected according to a Metropolis prescription with the effective Hamiltonian

$$
H=E+P L_{z}-N T \log \left(L_{z}\right)
$$




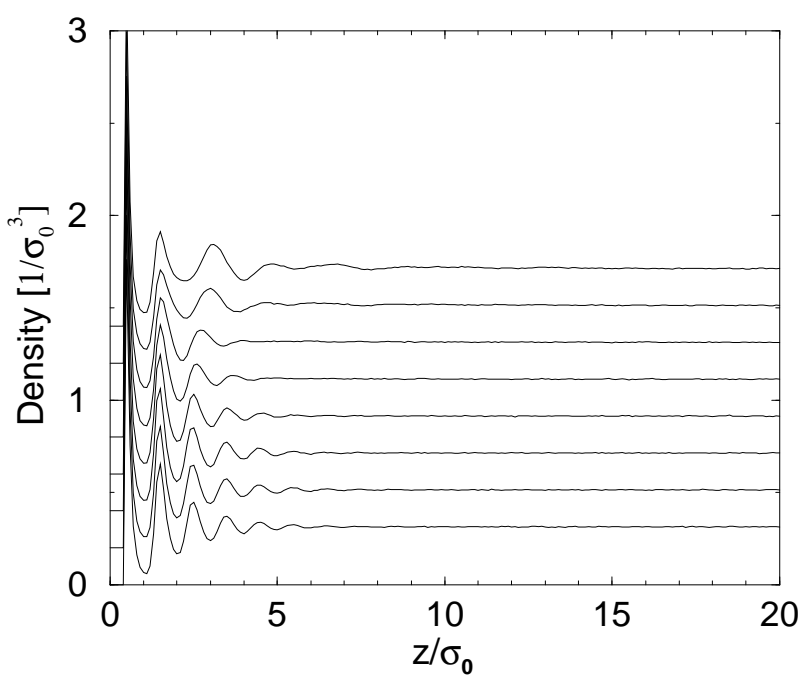

FIG. 1. Total density as a function of the distance $z$ from the wall for different grafting densities $\Sigma$. The curves are shifted by $0.2 / \sigma_{0}^{3}$ for better visibility. Grafting densities are (from top to bottom) $\Sigma \sigma_{0}^{2}=0.563,0.444,0.25,0.174,0.111$, $0.0625,0.0278,0$.

Here $E$ is the internal energy and $N$ the total number of ellipsoids in the system (solvent particles and chain monomers). In addition, we have also implemented special Configurational Bias Monte Carlo moves2324, which are specially adapted for our problem: The bonds of a randomly chosen chain were removed and redistributed such that a new chain was grown from the solvent. We calculated the probability $P_{\text {new }}$ of constructing this particular new chain, and the probability $P_{\text {old }}$ of just reconstructing the same old chain. The new chain was then accepted with the probability

$$
W_{\text {old } \rightarrow \text { new }}=\min \left(1, \frac{P_{\text {old }}}{P_{\text {new }}} \exp (-\Delta E / T)\right),
$$

where $\Delta E$ is the energy difference between the old and the new chain. Petails of this algorithm have been published elsewhere22. One "Monte Carlo step" consists on average of $N$ attempted translations, $2 \mathrm{~N}$ attempted rotations, one attempt of rescaling the box, and one configurational bias move.

Preliminary runs which started from initial configurations with particles oriented in the $z$ direction showed clearly that the walls align the solvent particles in a parallel way. The particles close to the walls turned around first, and the parallel orientation then propagated into the middle of the film. Unfortunately, this usually lead to a state where the director at the two walls pointed into two different directions, and was forced to twist around in the middle of the cell. In the later runs, we therefore prepared the configurations such that the particles all pointed into the $x$-direction initially. To this end, we applied a strong orienting field during roughly 50.000 Monte Carlo steps. After turning this field off, the system was equilibrated over at least 1 million Monte Carlo steps. Data were then collected over 5 million or more Monte Carlo steps.

Due to the complexity of the system, the calculations were very time consuming: Every run required several months on a Pentium III 800 processor. Therefore, we have not yet attempted to study larger systems. Unfortunately, this limits among other the maximum chain length - one has to ensure that a chain free "bulk region " remains in the middle of the cell. Simulations of systems with longer grafted chains will clearly be interesting in the future.

\section{RESULTS}

Figure 1 shows profiles of the total density (chain monomers and solvent particles) near the wall. They exhibit strong layering close to the wall, with decaying amplitude further away from the wall. The position of the first peak is independent of the grafting density. The distances between the other peaks increase with increasing grafting density. This is a first indication that the particle orientation changes from parallel to tilted as a function of the grafting density.

The total excess density at the wall,

$$
\rho_{\text {excess }}=\frac{1}{2} \int_{0}^{L_{z}} d z\left(\rho(z)-\rho_{\text {bulk }}\right)
$$

is shown as a function of the grafting density in Figure 2. Two opposite effects contribute: Particles are pushed towards the walls, but cannot move beyond $z=\sigma_{0} / 2$. At low grafting densities, the excluded volume at the wall is not quite compensated by the density excess in the density peaks close to the wall; at higher grafting densities, it is overcompensated. The overall excess density is small and rises roughly linearly with the grafting density.

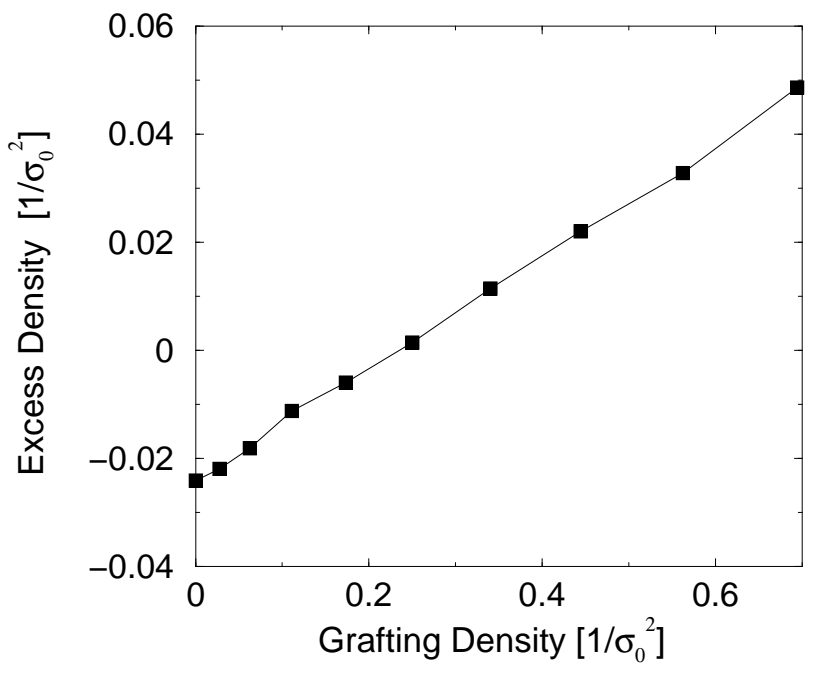

FIG. 2. Excess density vs. grafting density $\Sigma$. 


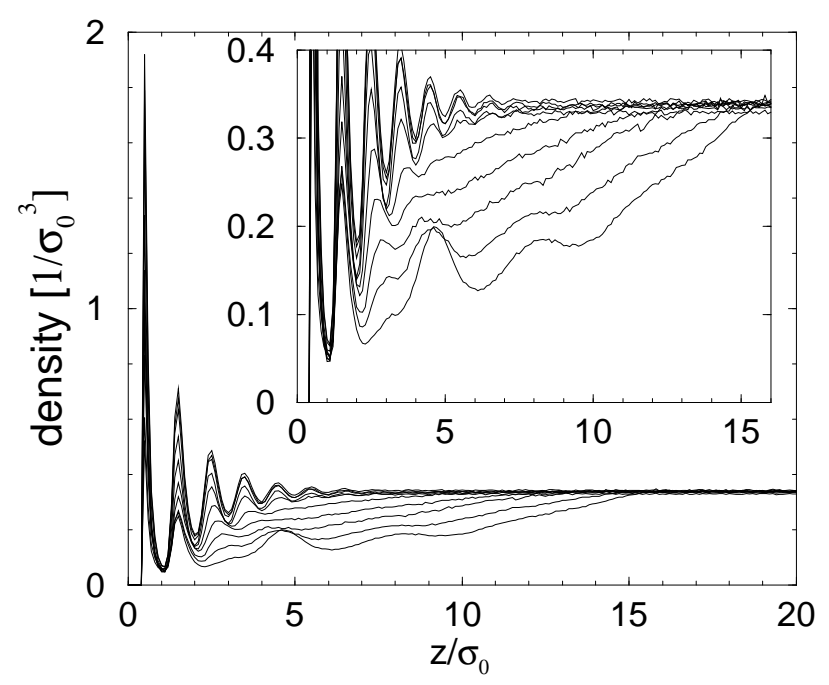

FIG. 3. Density of solvent particles as a function of the distance $z$ from the wall for different grafting densities: (from bottom to top) $\Sigma \sigma_{0}^{2}=0.563,0.444,0.34,0.25,0.174,0.111$, $0.0625,0.0278,0.00694,0$. Inset shows a blow-up of the same data.

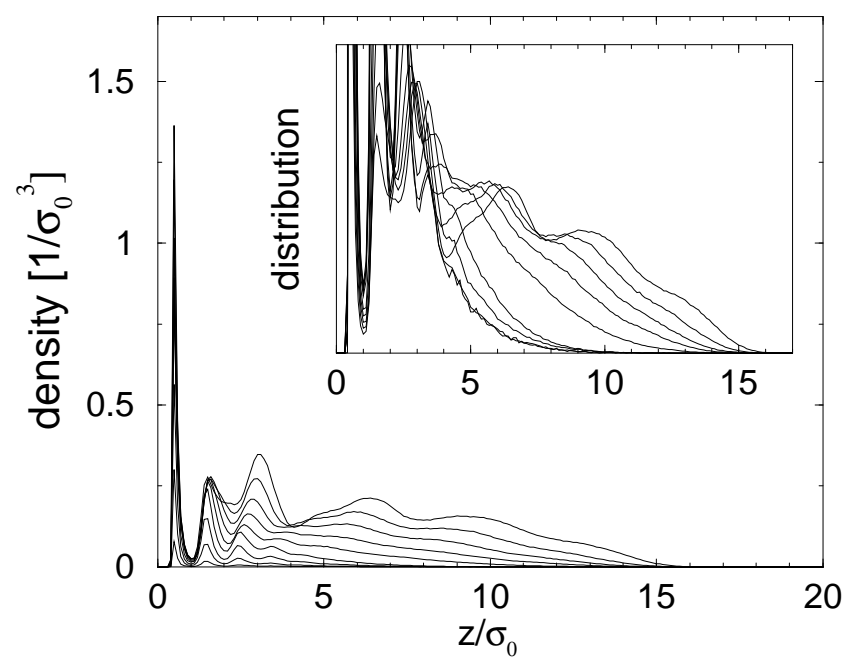

FIG. 4. Density of chain monomers as a function of the distance $z$ from the wall for different grafting densities: (from top to bottom) $\Sigma \sigma_{0}^{2}=0.563,0.444,0.34,0.25,0.174,0.111$, $0.0625,0.0278,0.00694$. Inset shows the chain density distributions, i. e., the normalized density profiles $\rho(z) / \int d z^{\prime} \rho\left(z^{\prime}\right)$.

It is instructive to analyze the profiles of solvent particles and chain monomers separately. The profiles of the solvent particles (Figure 3) show how the solvent particles are successively expelled from the chain region as the grafting density increases. The profiles of the chain particles are shown in Figure 1 . Close to the surface, they reproduce the layering of the overall density profile. The form of the profiles far from the surface depends on the grafting density: At low grafting densities, they decay smoothly; at higher grafting densities, they develop

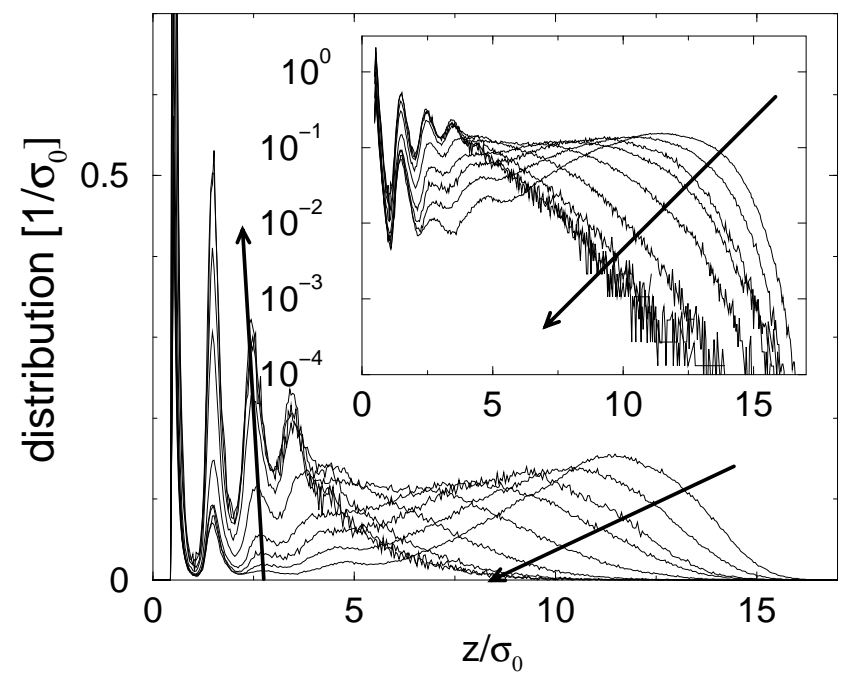

FIG. 5. Distribution of chain end monomers vs. distance $z$ from the surface for different grafting densities: (following the arrows) $\Sigma \sigma_{0}^{2}=0.563,0.444,0.34,0.25,0.174,0.111$, $0.0625,0.0278,0.00694$. Inset shows the same curves on a semi-logarithmic scale.

structure. Additional insight can be gained from looking at the normalized chain density distributions at different grafting densities $\Sigma$ (Figure 1 , inset). They are almost identical for the lowest $\Sigma$. As the grafting density increases, more and more chain monomers are pushed away from the surface, i. e., the chains stand up.

To study this in more detail, we inspect the distributions of chain ends in the $z$ direction (Figure 5). At low grafting densities, the chain end distributions again reproduce the layered structure of the nematic fluid close to the wall and decay smoothly, almost exponentially, far from the wall. At the grafting density $\Sigma / \sigma_{0}^{2} \sim 0.15$, a new broad peak emerges at some distance from the wall, which grows and moves away from the wall as the grafting density increases further. However, a fraction of chain ends still remains in the peaks close to the surface. Thus one observes two "types" of chains: Some that lie flat on the surface, and some that gradually stand up. Increasing the grafting density affects the conformations of the chains in two ways: The fraction of chains that lie flat decreases, and the chains that stand up extend deeper into the bulk.

Next we study the tilt angle $\theta$ of chains and particles with respect to the surface normal. Figure 6 shows the average angle $\langle\theta\rangle$ of the head-to-tail vector of chains and compares it with the corresponding value in a "dry" system of grafted chains without solvent particles. The dry layer is a reference system of randomly oriented chains, which are only subject to their mutual repulsive interactions and to the repulsive interaction with the wall. In the presence of solvent, the tilt angle of the chains is mostly higher than in the absence of solvent. At low 


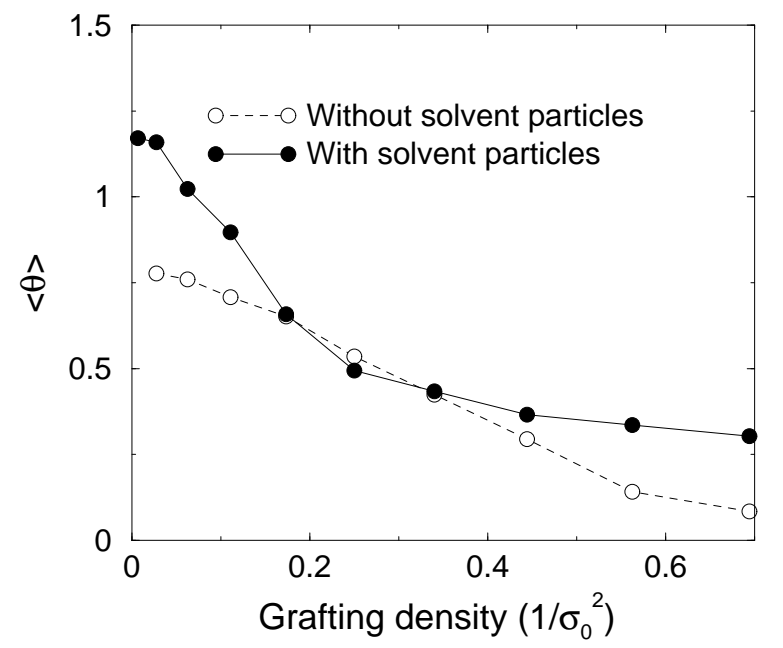

FIG. 6. Average tilt angle $\langle\theta\rangle$ of the head-to-end vector of the chains as a function of the grafting density $\Sigma$ without solvent (open symbols) and with solvent (filled symbols).

grafting densities, the nematic solvent determines the director profile close to the surface, and the chains lie down, i.e., $\theta$ is large. As the grafting density increases, the tilt angle drops rapidly and reaches that of the reference system at $\Sigma \sim 0.2 / \sigma_{0}^{2}$. Beyond $\Sigma \sim 0.3 / \sigma_{0}^{2}$, the tilt angle almost ceases to decrease. The chains retain tilt even at grafting densities where the chains in the dry system are almost perpendicular.

Compared to the dry system, the nematic solvent introduces a second important feature: It breaks the azimuthal symmetry. In the absence of solvent, the chains are disordered. In the presence of solvent, they tilt collectively in one direction. The direction of the tilt is related to the local direction of the director.

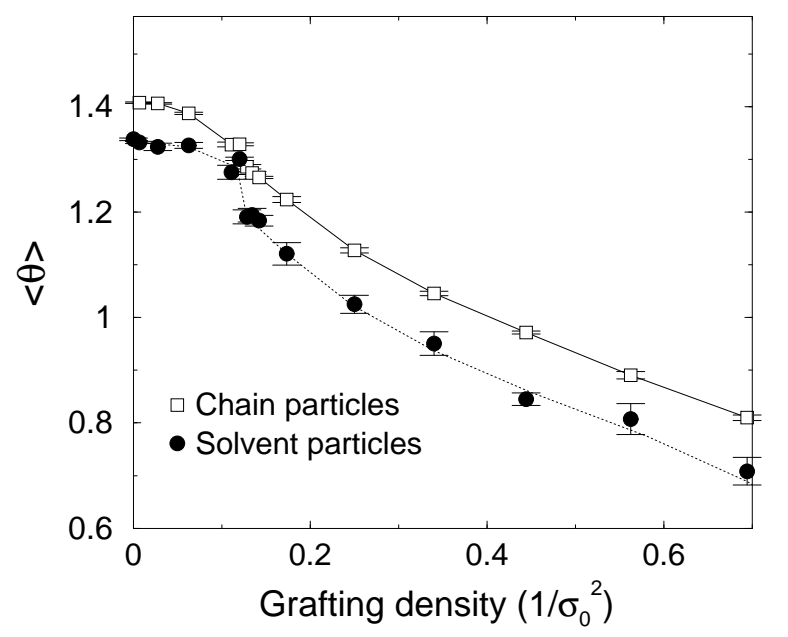

FIG. 7. Average tilt angle $\langle\theta\rangle$ of particles as a function of the grafting density $\Sigma$. Open symbols: Chain monomers, Filled symbols: solvent particles.
After having studied the tilt of whole chains, we discuss the tilt of individual particles. The average angle $\langle\theta\rangle$ for chain monomers and solvent particles is shown as a function of the grafting density in Figure 7 . The average tilt of chain monomers is consistently higher than that solvent particles, i. e., the chain region is more tilted than the bulk of the system. Looking at the average tilt of the solvent particles, one notices a a small jump at the grafting density $\Sigma^{*} \approx 0.13 / \sigma_{0}^{2}$. This suggests the presence of a first order phase transition. At grafting densities lower than $\Sigma^{*}$, the particles lie almost parallel to the wall. Beyond $\Sigma^{*}$, they exhibit tilt.

We can study these phenomena in more detail by inspecting the distribution $P(\cos (\theta))$ of tilt angles for solvent particles and chain particles, shown in in Figure 8. It is normalized such that the average number of particles in an angle window $[\theta, \theta+d \theta]$ is given by $N_{\theta, d \theta}=N_{\text {total }} P(\cos (\theta)) \sin (\theta) d \theta$. At low grafting densities, the distribution has always its maximum at $\theta=\pi / 2$, i.e., the particles lie flat at the wall. If one increases the grafting density, the distribution first broadens without shifting. At the grafting density $\Sigma \sim 0.13 / \sigma_{0}^{2}$, the maximum of the distribution detaches from $\pi / 2$ rather abruptly and starts moving to lower tilt angles. From then on, the maximum tilt angle decreases continuously with increasing grafting density. The chain monomer distribution has a small secondary maximum at the angle $\pi / 2$ at all grafting densities. This is the contribution of the fraction of chains which are flat on the substrate.

The maximum angle $\theta_{\max }$ is plotted as a function of the grafting density in Figure 9. The first order phase
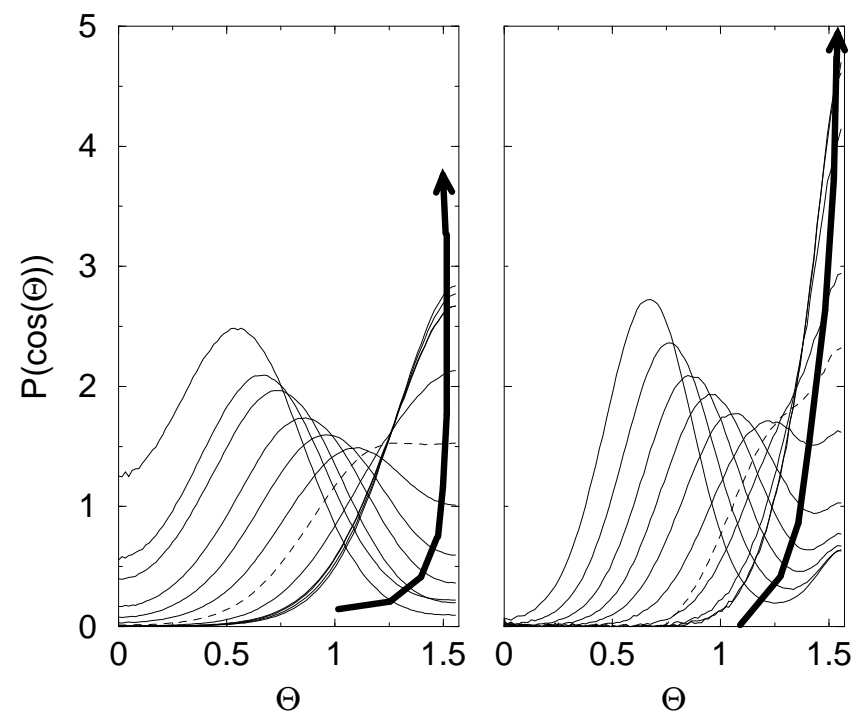

FIG. 8. Distribution $P(\cos \theta)$ of tilt angles for solvent particles (left) and chain monomers (right) and different grafting densities (following the arrows): $\Sigma \sigma_{0}^{2}=0.694,0.563,0.444$, $0.34,0.25,0.174,0.128,0.111,0.0625,0.0278,0.00694$, and 0 (only left figure). The distribution for $\Sigma \sigma_{0}^{2}=0.128$ is shown as dashed line. 


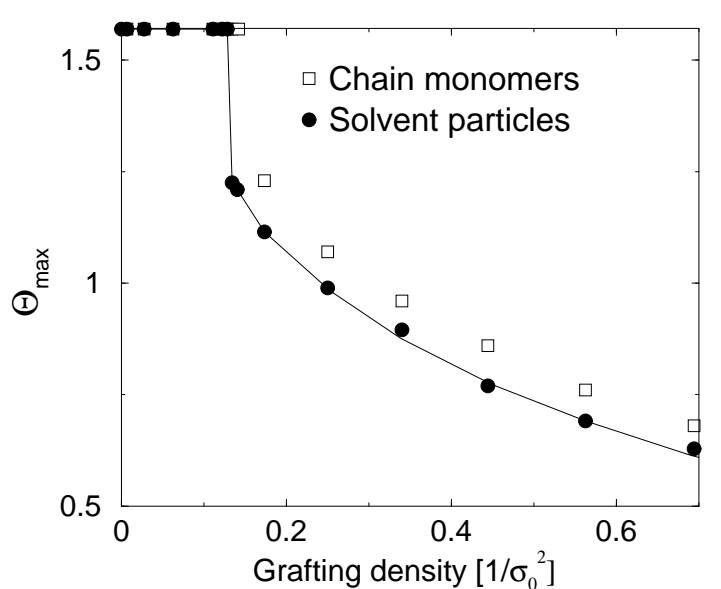

FIG. 9. Position of the maximum angle $\theta_{\max }$ vs. grafting density $\Sigma$ for chain particles (open squares) and solvent particles (filled circles).

transition in the nematic film becomes now very evident. The state of the film is best characterized by the angle distribution of the solvent particles, because the majority of them is outside of the chain layer. In contrast, the chain monomer distribution characterizes the structure close to the surface. The angle $\theta_{\max }$ for solvent particles jumps to a value different from $\pi / 2$ at $\Sigma^{*}=0.13 / \sigma_{0}^{2}$. In the case of the chain particles, the $\theta_{\max }$ jumps at a slightly higher grafting density $\left(\Sigma=0.17 / \sigma_{0}^{2}\right)$ : The chains which lie flat strengthen the peak at $\theta=\pi / 2$.

Finally, we turn to the discussion of the director profiles. The nematic order parameter and the nematic director in a slice of the simulation box are defined as follows: From the orientations $\mathbf{u}_{i}$ of all $n$ particles $i$ in the slice, one calculates the order tensor,

$$
\mathbf{Q}=\frac{1}{n} \sum_{i=1}^{n}\left(\frac{3}{2} \mathbf{u}_{i} \otimes \mathbf{u}_{i}-\frac{1}{2} \mathbf{I}\right)
$$

where $\mathbf{I}$ denotes the unity matrix and $\otimes$ the dyadic product. The largest eigenvalue of this matrix is the order parameter $S$ in the slice, and the corresponding eigenvector is the director $\mathbf{n}$. The order parameter in a bulk system with our parameters is roughly $S=0.75$. By construction, $S$ increases systematically if evaluated in subsystems with few particles. Therefore it tends to appear larger in a thin slice than in the bulk.

In order to calculate order parameter and director profiles, we cut the system into regular slices of thickness $\Delta z=1 \sigma_{0}$, starting from the left and the right wall up to almost the middle of the film. A thicker slice in the middle collected the remaining particles and absorbed the fluctuations of the box length $L_{z}$. This slice was then disregarded.
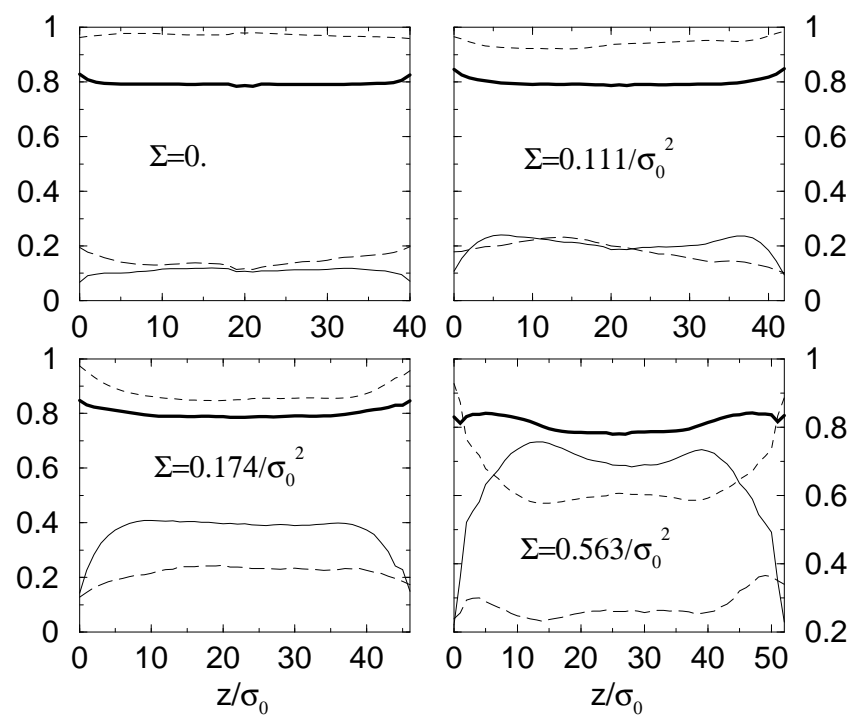

FIG. 10. Profiles of the order parameter $\langle S\rangle$ (thick lines) and the components of the director $\mathbf{n}$ (thin lines) vs. $z$ in the film for different grafting densities $\Sigma$ as indicated. thin solid line: $\left\langle\left|n_{z}\right|\right\rangle$; thin short dashed line: $\left\langle\left|n_{x}\right|\right\rangle$; thin long dashed line: $\left\langle\left|n_{y}\right|\right\rangle$.

Results for four grafting densities are shown in Figure 10. The order parameter $S$ varies very little throughout the film. In contrast, the director $\mathbf{n}$ changes quite dramatically at higher grafting densities: Close to the walls, it is always oriented parallel to the wall, $n_{z} \ll 1$. At low grafting densities, it stays parallel everywhere. At high grafting density, it turns around very rapidly within the first few $\sigma_{0}$ of the surface, and then continues to turn more slowly. Substantial reorientation takes place in a $z$-region of about the thickness of the chain layer (cf.

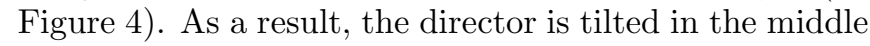
of the film.

The $y$-component of the director, $\left\langle\left|n_{y}\right|\right\rangle$, is overall smaller than the $x$-component. This is because the systems were initially prepared such that the director pointed in the $x$ direction. The diffusion time scale for reorientation without any driving force is much longer than the lengths of our runs (several million Monte Carlo steps). In one case, however, we happened to observe the onset of a reorientation - the system with grafting density $\Sigma=0.00694 / \sigma_{0}^{2}$ ventured into a configuration where the director twisted between the $x$ and the $y$ direction after 5 million Monte Carlo steps. The twist disappeared 2 million Monte Carlo steps later. It affected the profile of $\left\langle n_{z}\right\rangle$ only slightly.

Looking at Figure 10, one notices furthermore that the director profile hardly finds enough space to reach a plateau in the middle of the film at high grafting densities. This is a serious problem: Our systems of length $L_{z} \approx 50 \sigma_{0}\left( \pm 10 \sigma_{0}\right)$ are obviously too small to accommodate a real bulk region between the two surfaces. The results are subject to strong finite size effects, and any con- 
clusions about the anchoring behavior in a semi-infinite system must be viewed with caution. Additional finite size effects can be expected due to the limited lateral size of the simulation box, $L_{\|}=12 \sigma_{0}$. It would be desirable to study these effects by varying $L_{\|}$and $L_{z}$ systematically in different runs. Unfortunately, an extensive finite size analysis is computationally too expensive at the moment. Hence our quantitative results can be questioned. Nevertheless, we believe that the observed effects will persist qualitatively in semi-infinite systems.

These caveats stated, we collect the results for the order parameter $n_{z}$ in the middle of the film at different grafting densities (Figure 11). In a macroscopically thick film, it is directly related to the anchoring angle via $n_{z}=\cos \left(\theta_{\text {anchoring }}\right)$. Figure 11 confirms and completes the picture already suggested by Figures 7 and 9: At low grafting densities, the surface anchors the director parallel to the wall. The director component $\left\langle\left|n_{z}\right|\right\rangle$ remains constant and close to zero over a range of grafting densities, up to $\Sigma^{*}=0.13 / \sigma_{0}^{2}$. Then it jumps to a nonzero value and rises continuously thereafter. The transition from planar to tilted alignment is clearly discontinuous: A range of values $n_{z}$ is "forbidden". Hence a small range of anchoring angles $\theta_{\text {anchoring }}$ close to $\theta=\pi / 2$ $(\theta \in[0.4 \pi: 0.5 \pi])$ cannot by accessed at any grafting density. As mentioned above, finite size effects have to be expected. We cannot exclude the possibility that large scale fluctuations drive the transition to second order. But since the quantity most susceptible to large scale fluctuations in the tilted phase is presumably the azimuthal tilt angle, we suspect that the fluctuations will most likely reduce the azimuthal ordering and hence reduce the anchoring angle $\theta$. This implies that the gap in Figs. 9 widens, and that the transition becomes more strongly first order.

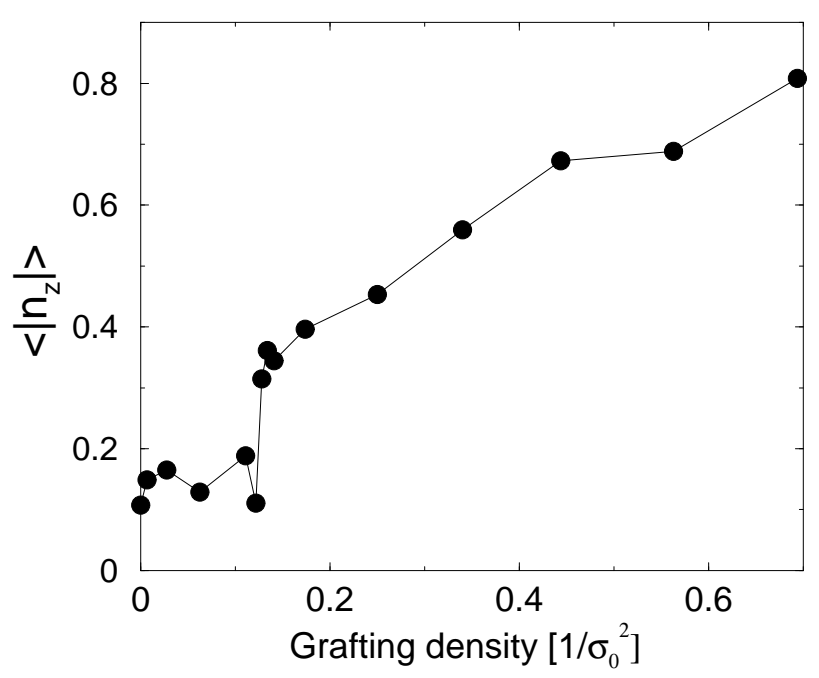

FIG. 11. $z$ component of the director in the middle of the film as a function of the grafting density.

\section{SUMMARY AND DISCUSSION}

In sum, we have studied the anchoring of a model nematic fluid on layers of liquid-crystalline chain molecules, grafted on a substrate which favors parallel anchoring. At low grafting densities, the substrate dominates and the nematic fluid is oriented parallel to the wall. A first order transition to a phase with tilted anchoring is encountered at a grafting density $\Sigma^{*}$. On increasing the grafting density further, the director gradually stands up. The phase transition goes along with a major reorganization within the chain layer: Below $\Sigma^{*}$, all chains lie flat at the wall. Above $\Sigma^{*}$, the chains separate into two distinct classes: Some remain flat at the wall, and some stand up. The tilt angle $\theta$ of the chains belonging to the latter group decreases with increasing grafting density, and the relative number of chains in that group increases.

The transition is very similar to the anchoring transition predicted by Halperin and Williams 17 : It mediates between a phase with parallel anchoring at low grafting density, $\Sigma<\Sigma^{*}$, and a tilted phase at higher grafting density, $\Sigma>\Sigma^{*}$. It is driven by a competition between the anchoring force from the substrate, which favors parallel anchoring, and the repulsive forces between the grafted chains, which force them to stand up at high grafting densities. However, the details of the underlying mechanisms are quite different. The theory of Halperin and Williams is devised for long chains with many hairpins, which can be treated as (anisotropic) entropic springs. Our chains are too short to support hairpins, and the configurational entropy is much less important. The other two constituents of the theory of Halperin and Williams, the excluded volume of the chains and the elastic energy of the nematic solvent, are obviously sufficient to bring about the phase transition.

The simulations and theories 1720 consider anchoring on layers of main-chain liquid crystalline chains. Experimentally, layers of grafted side-chain liquid crystalline polymers can be synthesized much more easily 12.13 . In these molecules, the liquid crystalline blocks orient themselves preferably perpendicular to the backbone of the chains. In order to generate a conflict between chain stretching and the anchoring on the substrate, one needs to prepare the substrate such that it aligns the liquid crystals in a homeotropic way. So far, this has not been done systematically, but prelimipary studies by Peng et al show that it should be feasible 13 . The question is: can we expect that an effect which has been predicted for main-chain liquid crystalline polymers will be observed in an experiment with side-chain liquid crystalline polymers?

Our study might help to answer this question. Comparing our simulation results with the theory of Halperin and Williams, we can conclude that the details of the chain conformations and the chain entropy do not really matter for the anchoring transition. It is robust enough to survive even in a regime for which the theory was never 
designed. Therefore the chances that it can be observed in real systems should be quite high.

\section{ACKNOWLEDGMENTS}

We have benefitted from useful conversations with A. Halperin, D. Johannsmann, J. Rühe, B. Peng, K. Binder, and in particular with M. P. Allen. We thank K. Binder and M. P. Allen for allowing us to perform simulations on the computers of their groups in Bristol and Mainz. This work was funded by the German Science Foundation (DFG).

${ }^{1}$ P.-G. de Gennes and J. Prost, The Physics of Liquid Crystals (Oxford University Press, Oxford, 1995).

${ }^{2}$ S. Chandrasekhar, Liquid Crystals (Cambridge University Press, Cambridge, 1992).

${ }^{3}$ B. Jerome, Rep. Progr. Phys. 54, 391 (1991).

${ }^{4}$ J. Cognard, Alignment of Liquid Crystals and their Mixtures (Gordon and Breach, London, 1982).

${ }^{5}$ N. L. Abbott, in Curr. Opn. in Coll. Interf. Sci. 2, 76 (1997).

${ }^{6}$ M. P. Allen, Mol. Phys. 96, 1391 (1999).

${ }^{7}$ B. Bahadur (edt.) Liquid crystals and uses, World Scientific, Singapore (1990).

${ }^{8}$ M. Schadt, Ann. Rev. Mater. Science, 27, 305 (1997).

${ }^{9}$ B.S. Scheuble, Kontakte 1, 34 (1989).

10 J. S. Patel, H. Yokoyama, Nature 362, 525 (1993).

${ }^{11}$ F. Benmouna, B. Peng, J. Rühe, D. Johannsmann, Liqu. Cryst. 26, 1655 (1999).

12 B. Peng, D. Johannsmann, J. Rühe, Macromolecules 32, 6759 (1999).

13 B. Peng, J. Rühe, D. Johannsmann, Adv. Mater. 12, 821 (2000).

${ }^{14}$ F. Benmouna, B. Peng, J. Gapinski, A. Patkowski, J. Rühe, D. Johannsmann, Liqu. Cryst. 28, 1353 (2001).

15 J. Rühe, Nachr. Chemie Techn. und Lab. 42, 1237 (1994).

${ }^{16}$ O. Prucker, J. Rühe, Macromolecules 31, 592 (1998); ibid, 602 (1998).

${ }^{17}$ A. Halperin, D. R. M. Williams, Europhys. Lett. 21, 575 (1993).

18 A. Halperin, D. R. M. Williams, J. Physics: Cond. Matt. 6, A297 (1994).

19 A. Halperin, D. R. M. Williams, Ann. Rev. of Mat. Science 26, 279 (1996).

${ }^{20}$ H. Lange, F. Schmid, Eur. J. Phys. E 7, 175 (2002).

${ }^{21}$ H. Lange, Dissertation Universität Mainz (2001).

${ }^{22}$ H. Lange, F. Schmid, Comp. Phys. Comm., in print (2002).

${ }^{23}$ M. N. Rosenbluth, A. W. Rosenbluth, J. Chem. Phys. 23, 356 (1955).

${ }^{24}$ D. Frenkel, B. Smit, Understanding Molecular Simulations, Academic Press, San Diego (1996).

${ }^{25}$ B. J. Berne and P. Pechukas, J. Chem. Phys. 56, 4213 (1975). 